\title{
Descending necrotizing mediastinitis after a trigger point injection
}

\section{Jae Young Choe', Jong Kun Kim², Dong Eun Lee ${ }^{2}$, Kang Suk Seo ${ }^{2}$, Jung Bae Park', Mi Jin Lee', Hyun Wook Ryoo², Jae Yun Ahn², Sungbae Moon ${ }^{2}$}

\author{
'Department of Pediatrics, Kyungpook National University School of Medicine, Daegu, Korea \\ ${ }^{2}$ Department of Emergency Medicine, Kyungpook National University School of Medicine, Daegu, Korea
}

Descending necrotizing mediastinitis (DNM) is a rare form of mediastinal infection. Most cases are associated with esophageal rupture. DNM after a trigger point injection in the upper trapezius has not been described previously. We present a case of DNM after a trigger point injection in the upper trapezius. A 70-year-old man visited the emergency department with chest discomfort and fever after a trigger point injection in the left upper trapezius. Chest computed tomography showed evidence of DNM, and antibiotic therapy was immediately administered intravenously. Because of the risk of sudden death, poor prognosis due to underlying disease, and his age, he declined surgical treatment and died of septic shock. Although trigger point injections are generally considered safe, caution should be used in patients with an underlying disease or in the elderly. Early diagnosis, broad-spectrum antibiotics, and aggressive surgical management are essential to improve the prognosis.

Keywords Mediastinitis; Injections; Intramuscular; Tomography, X-ray computed

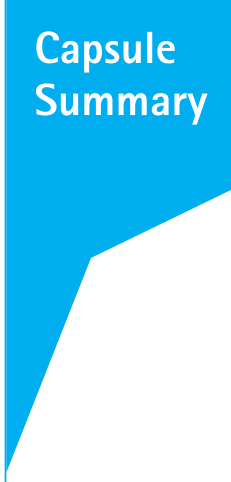

What is already known

Trigger point injections are generally considered safe. Descending necrotizing mediastinitis is a serious, life-threatening infection that can originate in the esophagus (through operation or rupture), or from oropharyngeal infection or cenvical trauma.

\section{What is new in the current study}

Although trigger point injections are generally safe, a complaint of chest discomfort or pain, fever, cough, and dyspnea following a trigger point injection in the neck area should prompt consideration of descending necrotizing mediastinitis.
Received: 5 March 2017

Revised: 7 July 2017

Accepted: 25 July 2017

Correspondence to: Dong Eun Lee Department of Emergency Medicine, Kyungpook National University School of Medicine, 680 Gukchaebosang-ro, Jung-gu, Daegu 41944, Korea E-mail: reveur2010@naver.com

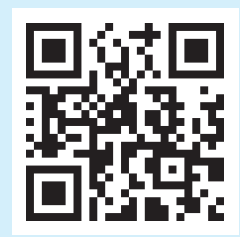

How to cite this article:

Choe JY, Kim JK, Lee DE, Seo KS, Park JB, Lee MJ, Ryoo HW, Ahn JY, Moon S. Descending necrotizing mediastinitis after a trigger point injection. Clin Exp Emerg Med 2017;4(3):182-185.

This is an Open Access article distributed under the terms of the Creative Commons Attribution Non-Commercial License (http:// creativecommons.org/licenses/by-nc/4.0/). 


\section{INTRODUCTION}

Mediastinitis is an inflammation of the connective tissues that involves mediastinal structures and fills the interpleural space. ${ }^{1}$ Descending necrotizing mediastinitis (DNM) is a serious, lifethreatening complication of mediastinal infection that can originate in the esophagus (through operation or rupture), or from oropharyngeal infection or cervical trauma. DNM is caused by the downward spread of a head and neck infection to the mediastinum through connecting deep and superficial cervical fascial planes. ${ }^{2}$ The risk factors for DNM include intravenous drug use, alcoholism, old age, and chronic debilitating comorbidities (diabetes mellitus, underlying malignancy, liver cirrhosis, and renal failure). ${ }^{3}$ DNM can be secondary to infectious or non-infectious causes and, depending on the etiology, can be acute or chronic. Diagnostic criteria for DNM were proposed by Estrera et al. ${ }^{4}$ in 1983: clinical manifestations of severe infection; characteristic radiographic features of mediastinitis; documentation of a necrotizing mediastinal infection during an operation, at autopsy, or both; and establishment of the relationship between DNM and an oropharyngeal or cervical infection. Despite advanced diagnostic and surgical techniques, DNM with sepsis reportedly has high rates of mortality, primarily because of delayed diagnosis. ${ }^{5}$ However, the diagnosis of DNM can be difficult, owing to the vagueness of the symptoms. ${ }^{6}$ DNM after a trigger point injection (TPI) has not been described previously; here, we present a case of DNM after TPI in the left upper trapezius.

\section{CASE REPORT}

A 70-year-old Asian man with diabetes mellitus treated with oral hypoglycemic agents and a history of myocardial infarction managed with percutaneous coronary intervention and antiplatelet drugs visited the emergency department (ED) of our hospital complaining of 7 days of progressive chest discomfort, fever, and chills. Ten days prior, he visited a local clinic with left upper back and shoulder pain. He was diagnosed with myofascial pain syndrome and received a TPI with lidocaine using a 21-gauge, 2.0inch needle in the left upper trapezius. Three days after the injection, he complained of substernal chest pain of a squeezing nature and chills, and visited the ED of another hospital. Coronary angiography at that hospital was normal. His symptoms progressively worsened despite administration of analgesics, and he was transferred to our hospital.

He was an active smoker, with a cumulative exposure of 35 pack-years, and a heavy drinker, consuming 1 bottle of alcohol daily. He did not have clinical evidence of a recent head and neck

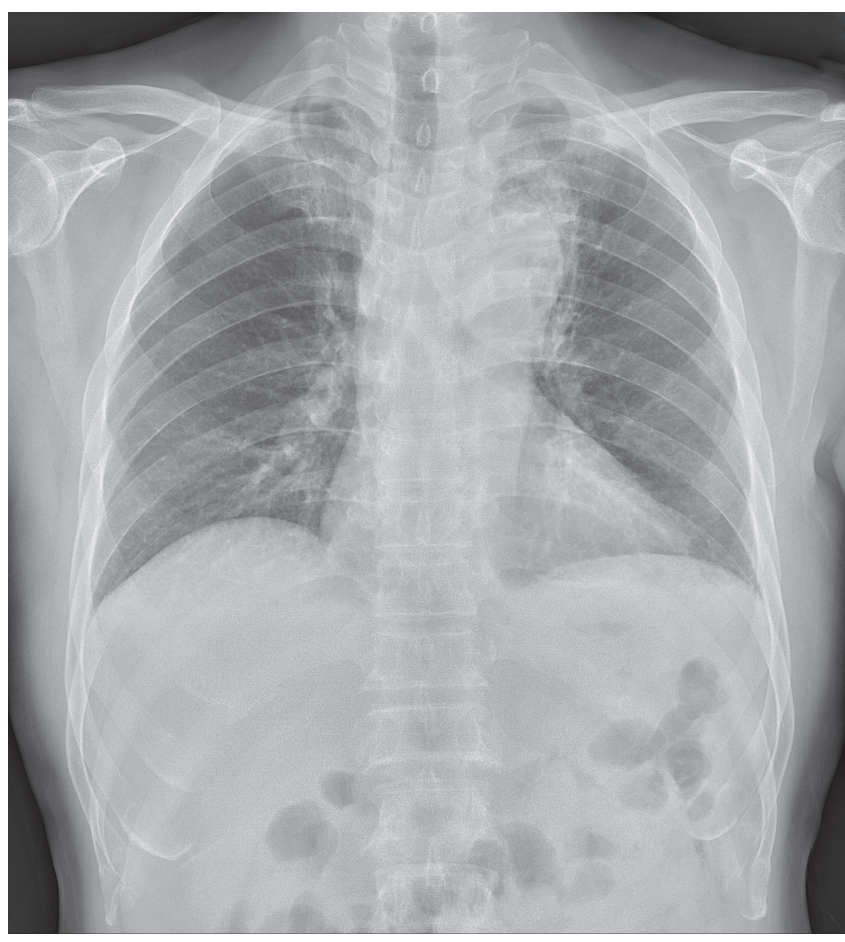

Fig. 1. Chest radiography. Consolidation is seen in the left upper lobe of the lung.

infection or trauma. He was $163 \mathrm{~cm}$ tall and weighed $60.3 \mathrm{~kg}$ (body mass index, $22.7 \mathrm{~kg} / \mathrm{m}^{2}$ ). In the ED, his blood pressure was 120/78 $\mathrm{mmHg}$, heart rate was 110 beats/min, oxygen saturation was $97 \%$, and axillary temperature was $38.2^{\circ} \mathrm{C}$. A physical examination revealed tenderness and redness from the left side of the upper sternum to the same side of the shoulder. The other examination results were normal.

Laboratory data were significant for a white blood cell count of $19.9 \times 10^{9} / L$, with $86.7 \%$ neutrophils. Both C-reactive protein and erythrocyte sedimentation rate were elevated, at $26.5 \mathrm{mg} / \mathrm{L}$ (reference, $<0.5 \mathrm{mg} / \mathrm{dL}$ ) and $80 \mathrm{~mm} / \mathrm{hr}(0-10 \mathrm{~mm} / \mathrm{hr})$, respectively. Arterial blood gas analysis showed pH 7.48, $\mathrm{PCO}_{2} 28 \mathrm{mmHg}$ $\mathrm{PO}_{2} 115.3 \mathrm{mmHg}$, and $\mathrm{HCO}_{3} 20.9 \mathrm{mmol} / \mathrm{L}$. Serum glucose was 249 $\mathrm{mg} / \mathrm{dL}$ and a urine test strip was 4+ for glucose. Other laboratory test results were normal, including plasma levels of electrolytes, troponin, and creatinine kinase-MB. Aerobic and anaerobic blood cultures were drawn before empirical antibiotic treatment.

Chest radiography showed upper mediastinal widening, protrusion of the aortic arch, and consolidation in the left upper lobe (Fig. 1). Contrast-enhanced chest computed tomography (CT) revealed a $3.8 \times 3-\mathrm{cm}$ outpouching low-density lesion with an infiltrative margin arising from the aortic arch, diffuse mild wallthickening in the aorta, and an incidental $6.8-\mathrm{cm}$ heterogeneous enhancing mass in the right hepatic lobe (Fig. 2). Magnetic reso- 

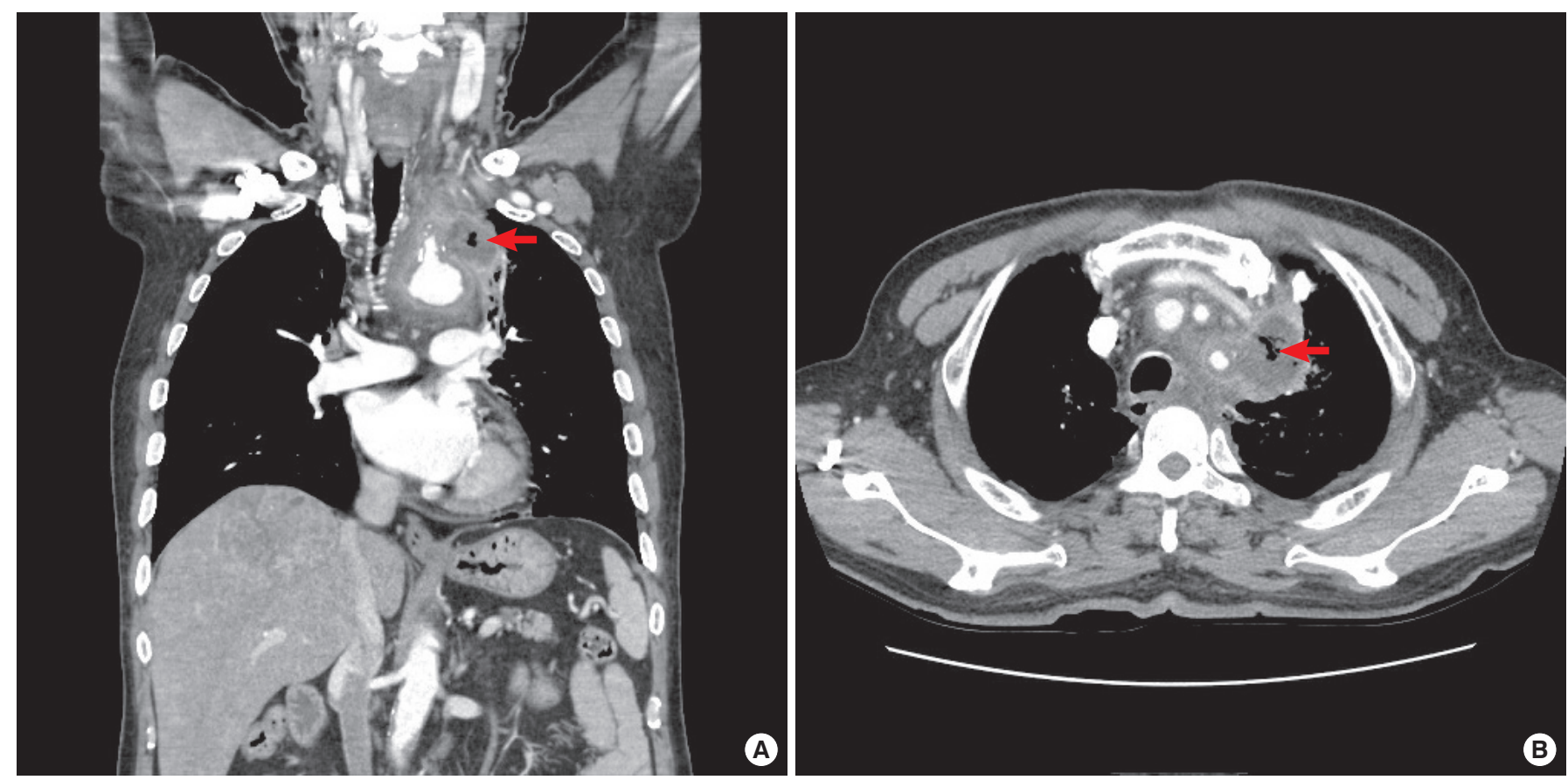

Fig. 2. (A) Contrast-enhanced chest computed tomography (CT, coronal plane). (B) Contrast-enhanced chest CT (axial plane). Chest CT showing well-defined, lobulated fluid collections with gas bubbles in the aortic arch (arrow).

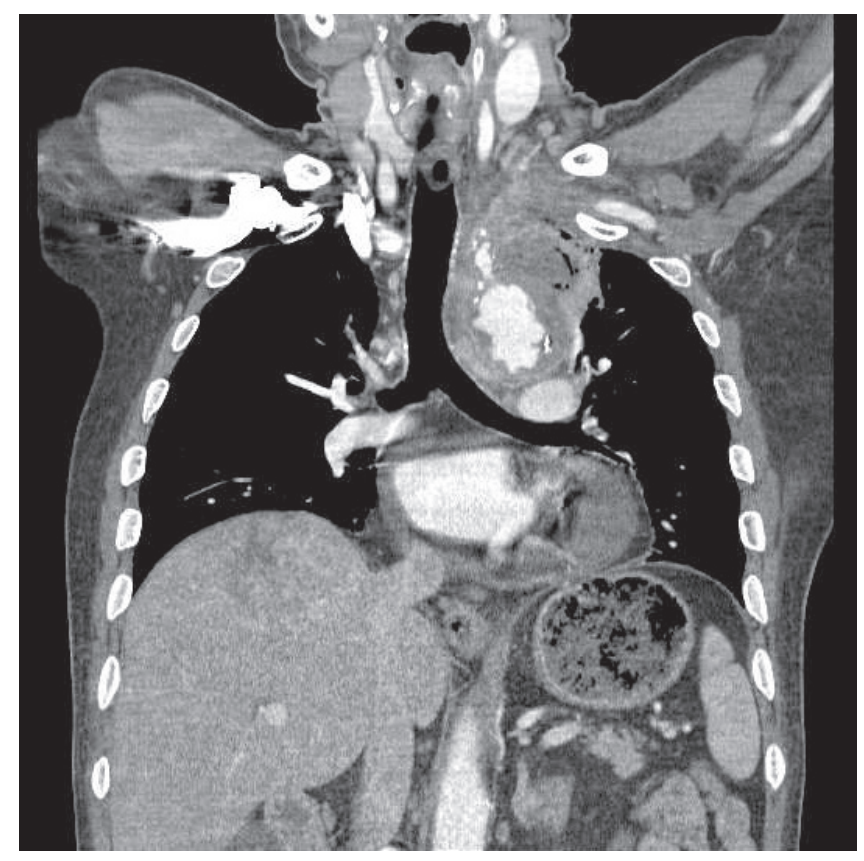

Fig. 3. Follow-up chest computed tomography after 8 days. Increased mediastinal infiltration and a low-density lesion with an air bubble are visible.

nance imaging of the liver suggested hepatocellular carcinoma in the $\mathrm{S} 7$ segment.

He was treated immediately with intravenous ceftriaxone and metronidazole, and the initial blood culture revealed Staphylo- coccus aureus. Based on clinical and radiological features, he was diagnosed with DNM after TPI.

Fever persisted following the ED visit, and both C-reactive protein and erythrocyte sedimentation rate remained elevated, at $23.6 \mathrm{mg} / \mathrm{L}$ and $37 \mathrm{~mm} / \mathrm{hr}$, respectively. Antibiotics were changed on hospital day 3 to piperacillin/tazobactam. However, intermittent fever persisted until hospital day 5 , and finally subsided when the antibiotics were changed to meropenem and vancomycin.

On hospital day 9, he had hemoptysis. Follow-up CT showed increased mediastinal infiltration and a low-density lesion with an air bubble and a penetrating atherosclerotic ulcer at the aortic arch (Fig. 3). Based on the CT, we explained the process of surgical drainage. However, he and his family refused aggressive management such as surgery, because of the risk of sudden death during the operation, poor prognosis of underlying hepatocellular carcinoma, and his age. Despite antibiotic therapy, his general condition worsened, and he authorized a do-not-resuscitate order. On hospital day 30, he died because of septic shock.

\section{DISCUSSION}

The majority of DNM cases are associated with cardiovascular operations, esophageal perforation, tracheobronchial perforation, mediastinal extension of pulmonary infections, or mediastinal extension of head and neck infections. However, to the best of our knowledge, DNM following TPI in the upper trapezius has not 
been described.

Trigger points are focal hyperirritable areas within a taut band of skeletal muscle that are painful on precise compression. Trigger points can be very painful and disabling and even cause weakness. The rhomboid and trapezius muscles, located in the upper back and shoulder areas, are common sites of trigger points. ${ }^{7}$ A TPI is one of the most effective therapeutic approaches, and is generally considered very safe. The most common complication is infection, including osteomyelitis, epidural abscess, and necrotizing fasciitis. Noninfectious complications include peripheral nerve injuries, pneumothorax, air embolism, and pain or swelling at the site of the injection. ${ }^{8}$

The diagnosis of DNM is often difficult due to nonspecific and vague early symptoms, which include chills, fever, tachycardia, dyspnea, and a nonproductive cough. ${ }^{2,3,5}$ A CT scan can immediately confirm mediastinitis with high accuracy, showing soft tissue infiltration with loss of the normal fat planes or collection of fluid with or without the presence of gas bubbles. The use of contrast-enhanced chest CT is the diagnostic method of choice for patients in whom DNM is suspected. ${ }^{2-4}$

Treatment for DNM includes broad-spectrum antibiotics and early aggressive surgical intervention. Intravenous broad-spectrum antibiotic therapy is not effective without adequate surgical drainage of cervical and mediastinal collections and extensive debridement and excision of necrotic tissue. In reported cases of DNM, successful surgical management involves a combination of cervical and mediastinal drainage, with or without open thoracotomy. ${ }^{1,3,4,9,10}$ More recently, less invasive approaches that successfully employ thoracoscopic or mediastinoscopic drainage have been reported. ${ }^{11,12}$

The present case highlights the importance of collecting the patient history, physical examination, and radiographic findings to differentiate between DNM and other causes that present similar symptoms, including chest pain. Although TPI is generally safe, a complaint of chest discomfort or pain, fever, cough, and dyspnea following a TPI in the neck area should prompt consideration of DNM. A high index of suspicion and appropriate investigations are important, as a favorable outcome depends on early diagnosis and surgical intervention. In summary, early diagnosis, aggressive medical and surgical interventions, and close surveillance with serial CT are crucial for reducing the mortality associated with DNM.

\section{CONFLICT OF INTEREST}

No potential conflict of interest relevant to this article was reported.

\section{REFERENCES}

1. Mihos P, Potaris K, Gakidis I, Papadakis D, Rallis G. Management of descending necrotizing mediastinitis. J Oral Maxillofac Surg 2004;62:966-72.

2. Ridder GJ, Maier W, Kinzer S, Teszler CB, Boedeker CC, Pfeiffer $J$ J. Descending necrotizing mediastinitis: contemporary trends in etiology, diagnosis, management, and outcome. Ann Surg 2010;251:528-34.

3. Sumi Y. Descending necrotizing mediastinitis: 5 years of published data in Japan. Acute Med Surg 2015;2:1-12.

4. Estrera AS, Landay MJ, Grisham JM, Sinn DP, Platt MR. Descending necrotizing mediastinitis. Surg Gynecol Obstet 1983; 157:545-52.

5. Sarna T, Sengupta T, Miloro M, Kolokythas A. Cervical necrotizing fasciitis with descending mediastinitis: literature review and case report. J Oral Maxillofac Surg 2012;70:1342-50.

6. Sandner A, Borgermann J, Kosling S, Silber RE, Bloching MB. Descending necrotizing mediastinitis: early detection and radical surgery are crucial. J Oral Maxillofac Surg 2007;65: 794-800.

7. Wyant GM. Chronic pain syndromes and their treatment. II. Trigger points. Can Anaesth Soc J 1979;26:216-9.

8. Cheng J, Abdi S. Complications of joint, tendon, and muscle injections. Tech Reg Anesth Pain Manag 2007;11:141-7.

9. Kim JT, Kim KH, Lee SW, Sun K. Descending necrotizing mediastinitis: mediastinal drainage with or without thoracotomy. Thorac Cardiovasc Surg 1999;47:333-5.

10. Roccia F, Pecorari GC, Oliaro A, et al. Ten years of descending necrotizing mediastinitis: management of 23 cases. J Oral Maxillofac Surg 2007;65:1716-24.

11. Roberts JR, Smythe WR, Weber RW, Lanutti M, Rosengard BR, Kaiser LR. Thoracoscopic management of descending necrotizing mediastinitis. Chest 1997;112:850-4.

12. Son HS, Cho JH, Park SM, Sun K, Kim KT, Lee SH. Management of descending necrotizing mediastinitis using minimally invasive video-assisted thoracoscopic surgery. Surg Laparosc Endosc Percutan Tech 2006;16:379-82. 